\title{
CHROMATOGRAPHY-BASED PARTIAL PURIFICATION AND CHARACTERIZATION OF ANTIMICROBIAL COMPOUND ISOLATED FROM A TERRESTRIAL STREPTOMYCES
}

\author{
SUDHA SRI KESAVAN S*, USHA NANDHINI S, GIRIJA VASUDEVAN
}

Department of Biotechnology, Sathyabama University, Jeppiaar Nagar, Rajiv Gandhi Salai, Chennai - 600 119, Tamil Nadu, India.

\author{
Email:sudhak7phd@gmail.com
}

Received: 11 September 2017, Revised and Accepted: 27 October 2017

ABSTRACT

Objectives: A potent Streptomyces strain isolated from garden soil samples of Sathyabama University, Chennai, and the characterization and its antimicrobial activity was reported previously. This report deals with partial purification and characterization of the partially purified antimicrobial compound by liquid chromatography-mass spectrum (LC-MS) method.

\begin{abstract}
Method: The crude extract was characterized by ultra violet-visible spectrum (UV) and Fourier transform infrared spectroscopy (FTIR) subsequently partial purification was done by thin-layer chromatography, and the bands were used for LC-MS analysis. The obtained mass spectrum was searched in NIST for species data by molecular weight. The compound was identified by comparing the mass spectrum with the known compounds mass spectrum having 98-100\% identical. The biological activity of the identified compound was searched in PubChem.
\end{abstract}

Results: The UV and FTIR spectrum indicate the presence of hydroxyl group, aliphatic chain, aromatic, aliphatic amines, and ethers in the crude extract. The LC-MS spectrum revealed the presence of 12 compounds in high intensity resembling antimicrobial compounds such as imidazole, $\mathrm{N}$-trifluoroacetyl, cyclohexane, 2-nitro, and stigmasterol.

Conclusion: The instrumental analysis shows the presence of 14 different compounds, and PubChem compound search shows these compounds having various biological activities including antibacterial, antifungal, anticancer, antitumor, and antidepressant. This report insists us further to evaluate the anticancer, antitumor, and antitrypanosomal activities of the partially purified compound.

Keywords: Terrestrial Streptomyces, Antimicrobial compound, Characterization, Liquid chromatography-mass spectrrum, Fourier-transform infrared spectroscopy.

(c) 2018 The Authors. Published by Innovare Academic Sciences Pvt Ltd. This is an open access article under the CC BY license (http://creativecommons. org/licenses/by/4. 0/) DOI: http://dx.doi.org/10.22159/ajpcr.2018.v11i22501

\section{INTRODUCTION}

The rapid emergence of drug resistance among pathogenic bacteria, especially multidrug-resistant bacteria, underlines the need to look for new antibiotics $[1,2]$. Filamentous actinomycetes are known to have the ability to produce a wide variety of secondary metabolites [3,4], many of which have useful applications in human and veterinary medicine and agriculture [5], such as enzyme immuno-modulators, antibiotics, insecticides, herbicides, and anticancer agents [6]. Among Actinomycetes, Streptomyces which has long been recognized as a major source of bioactive molecules covers around $70 \%$ of the total antibiotic products $[7,8]$. This genus was confirmed to be promising bacteria against several pathogens and is well known for their potential to produce a large number of inhibitory metabolites used in industry and pharmacy [9].

Actinomycetes are widely distributed and are next to bacteria in the order of abundance in soil [10]. In the past two decades, there has been a decline in the discovery of new lead compounds from common soilderived actinomycetes [11]. The diverse climatic and soil conditions support the existence of diverse species of actinomycetes which may prove to be a potential source for effective metabolites active against major pathogens. Such molecules, if found and identified, would be utilized to formulate new antibiotics. Hence, the present study made an attempt to estimate the actinomycetes populations in soil (the rhizosphere of plants) of Sathyabama University, so as to screen for their antimicrobial properties.

\section{MATERIALS AND METHODS}

Isolation, screening, and crude extract production from potent actinomycetes

The materials and methods followed for isolation, antimicrobial screening, and crude extract production were reported previously by Sudha et al., 2015 [12].

Characterization of active compound in the crude extract Ultra violet (UV)-visible spectrum analysis

An evaluation of the UV-spectrum of the microbial extracts was carried out to determine the chromophores present in the metabolites. Optical measurements were performed on UV-visible spectrophotometer SHIMADZU model UV-160A. The spectrum was scanned over the desired wavelength range of $200-400 \mathrm{~nm}$.

Fourier-transform infrared spectroscopy (FT-IR) spectrum analysis The Fourier-transform infrared spectrum of column active fraction was analyzed to identify the functional groups present in the active compounds [13]. Electromagnetic radiation ranging between $2500 \mathrm{~nm}$ and $20,000 \mathrm{~nm}$ is passed through a sample and is absorbed by the bands of the molecules in the sample causing them to stretching or bending. The wavelength of the radiation absorbed is characteristic of the bond absorbing it. IR spectrum was recorded on a Bruker FT-IR instrument equipped with AT-XT Golden gate accessories. The spectrum was scanned in the $400-4000 / \mathrm{cm}$ range. The spectrum was plotted as percentage transmittance versus wave number. The spectra obtained 
through those samples were compared and interpreted for the shifting of functional peaks.

\section{Thin-layer chromatography (TLC)}

Ethyl acetate crude extract was subjected to TLC using ethyl acetate:hexane $(7: 3)$ ratio and benzene: hexane:ethyl acetate:methanol (1:1:1:1). The developed plate was visualized under UV at $366 \mathrm{~nm}$. The distinct bands were visualized, and the resolving factor (Rf) value was determined by the following equation:

\section{$\mathrm{Rf}=\frac{\text { Distance traveled by the solute }}{\text { Distance traveled by the solvent }}$}

The TLC was performed repeatedly, and the bands were collected along with the silica and centrifuged to separate the active compounds from the supernatant. The collected supernatant was allowed for dryness and used for liquid chromatography-mass spectrrum (LC-MS) analysis.

\section{$L C-M S$}

Determination of the bioactive compound by LC/MS analyses was performed on Shimadzu LCMS-2020 system (ESI-Single Quad) equipped with an positive ionization source (ESI). The LC Pump system was performed at $27^{\circ} \mathrm{C}$ using a Zorbas column $(250 \times 4.6 \mathrm{~mm}$; C18, $5 \mathrm{~mm}$ particle size). Samples were injected using High Performance autosampler with a $10 \mu \mathrm{L}$ injection volume. Separations were carried out by using Acetonitrile $0.01 \% 0.01 \%$ formic acid and water with the flow rate 300 microlitre per minute throughout the run. Full mass scan spectra were recorded over a range of 50-1000 m/z.

\section{RESULTS}

The earlier report of Sudha et al., 2015 [12], described the antimicrobial potential of the selected isolate BN8 and its crude extract. The crude extract inhibited the growth of both bacterial and fungal strains with an MIC $=50 \mu \mathrm{g} / \mathrm{ml}$ against Pseudomonas aeruginosa, $25 \mu \mathrm{g} / \mathrm{ml}$ against Staphylococcus aureus, Klebsiella pneumonia, and Bacillus cereus. The MIC value of the crude extract against the bacterium E. coli was $12.5 \mu \mathrm{g} / \mathrm{ml}$. The MIC of $12.5 \mu \mathrm{g} / \mathrm{ml}$ was observed in the crude extract against A. flavus, $25 \mu \mathrm{g} / \mathrm{ml}$ against Aspergillus niger, and $50 \mu \mathrm{g} / \mathrm{ml}$ against Aspergillus fumigatus, respectively.

\section{Characterization of crude extract}

\section{UV-visible spectrum analysis}

Many microbial metabolites have characteristic absorption spectra in the ultraviolet visible region; such spectra can be used for initial identification by comparison with spectral libraries to determine if the compound belongs to a particular chemical class, for identifying components related to a known structure and for monitoring the production of secondary metabolites [14,15]. UV spectra of the crude extract of the isolate BN 8 showed the absorbance maximum peaks at 270 and $300 \mathrm{~nm}$ (Fig. 1).

\section{Infrared spectroscopy}

FT-IR spectrum of the crude extract showed that a high absorption bands at $1020.31 / \mathrm{cm}$ represent the $\mathrm{C}-\mathrm{H}$ in plane bending ${ }^{1}$ and $3196.05 / \mathrm{cm}$ indicate hydroxyl group. The absorption bands at $2328.08 / \mathrm{cm}$ and $2918.30 / \mathrm{cmcorresponding}$ to C-H stretch correspond to aliphatic chain. While the double bond of polygenic compound seen at $1622.13 / \mathrm{cm}$, the bands observed at 1408 was assigned to the C-N stretching vibrations of aromatic and aliphatic amines, respectively. The band at 1072.42/cm corresponds to ethers (C-O-C) (Fig. 2).

$T L C$

TLC analysis of the ethyl acetate extract of isolate BN 8 revealed the presence of active compounds that were visualized in UV and iodine spray. On the combination of benzene: hexane:ethyl acetate:methanol in 1:1:1:1 ratio to separate the active principle present in the crude extract, three separated bands with the $\mathrm{Rf}=4.5,4.4$, and 2.4 were obtained under UV and also iodine spray. When the sample is separated by the solvent system of ethyl acetate:hexane in 7:3 ratio, also three separated bands with the $\mathrm{Rf}=4.8 \mathrm{~cm}, 3.7 \mathrm{~cm}$, and $3.2 \mathrm{~cm}$ were obtained under UV (Fig. 3a and b).

\section{LC-MS}

LC-MS analysis of the partially purified compound shows the presence of 14 dominant compounds. The mass spectrum was used to determine the chemical structure by NIST for species data by molecular weight. The compound was identified by comparing the mass spectrum with the known compounds mass spectrum having $98-100 \%$ identical. The biological activity of each compound was determined using PubChem

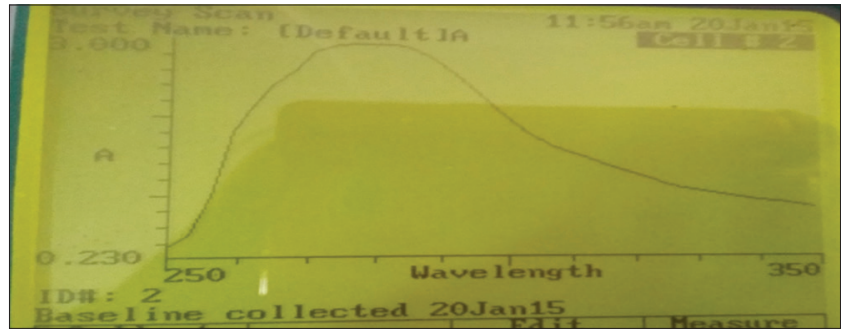

Fig. 1: Ultraviolet absorption spectrum of the ethyl acetate extract of selected isolate BN 8

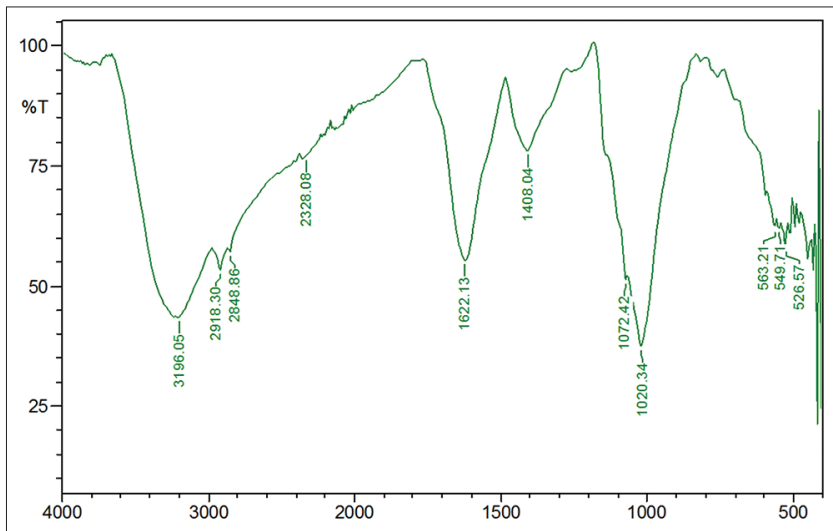

Fig. 2: Fourier-transform infrared spectroscopy spectrum of crude extract produced by isolate BN 8

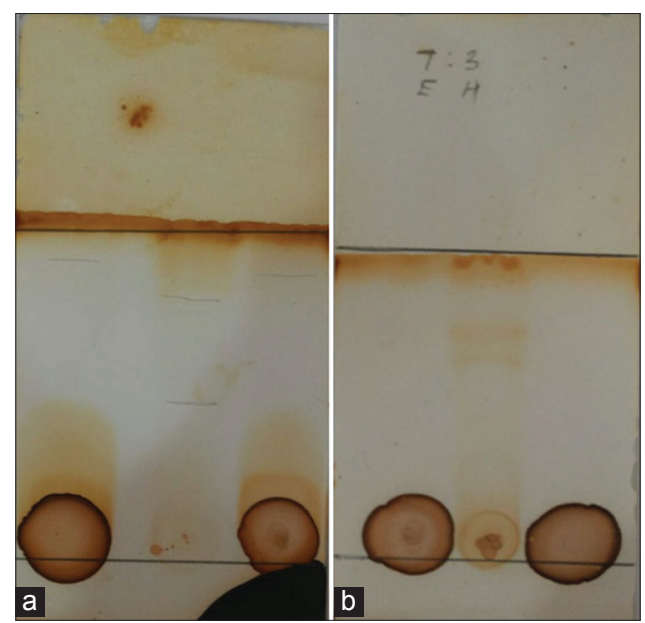

Fig. 3: (a) Separated bands on thin-layer chromatography (TLC) (benzene:hexane:ethyl acetate:methanol [1:1:1:1]). (b) Separated bands on TLC (ethyl acetate:hexane [7:3]) 
search. All the individual mass spectrum resembling compounds were identified, and their biological activities were summarized in Table 1.

\section{DISCUSSION}

In the present study, screening of terrestrial actinobacteria against bacterial and fungal pathogens resulted for the isolation of potential Streptomyces sp. Similarly, the actinobacteria isolated from marine sediments exhibited antibacterial activity against selected drugresistant pathogens [16]. Slavica et al. [17] reported that the UV spectral data for the ethyl acetate extract of selected fermented broth showed the maximum absorbance peaks ranging between 200 and $295 \mathrm{~nm}$ and the characteristics of absorption peaks indicated a highly polyene nature.

The FTIR spectrum of Streptomyces hygroscopicus BDUS 49 compound showed two absorption peaks in the region of 3500 and $1730 / \mathrm{cm}$. The spectrum indicates that the compound had $\mathrm{OH}$ and possibly lactone carbonyl group. Another two absorption peaks showed in the region of 1700 (carbonyl) and between 1610 and $1630 / \mathrm{cm}$. This peak indicates that the compound had carbonyl and alkenes $(\mathrm{C}=\mathrm{C})$ group [18].

Antifungal activity of TLC fraction with the $\mathrm{R}_{\mathrm{f}}=0.39$ [19] and similarly the $\mathrm{R}_{\mathrm{f}}$ value of the compound produced by Streptomyces sp. VPTSA18 was 0.48 by TLC chromatogram. The color of the separated antimicrobial compound was reddish brown [20].

The LC-MS analysis shows that the partially purified antimicrobial compound contains 12 components in high density. The PubChem chemical search shows that the compound 3-nitrobenzenesulfonamid is an anticancer compound. The 5-nitrofuran-2-carboxylic acid exhibited cytotoxicity toward human MRC-5 cells. The compound 2-methylthiazole was active against acute lymphoblastic leukemia.

Cyclohexane, 2-nitro an antibacterial active [21] compound presence also observed (Fig. 4). Imidazole, N-trifluoroacetyl an antibacterial and antifungal [22] compound also found by comparing the mass spectrum of compounds from NIST (Figs. 5 and 6). The presence of compounds such as thiazolidine, butanamide, $\mathrm{N}$-(4-chlorophenyl)-3oxo-, 4,4'-oxydiphthalic dianhydride, cyclohexanol, 2-bromo-, cis- with anticancer, antitumor, and cytotoxic activity were detected.

The partially purified compound shows the presence of stigmasterol with antitrypanosomal activity against Trypanosoma brucei an antibacterial compound. Rokade and Sayyed [23] reported that 1-naphthalene carboxamide is an effective antimicrobial agent. The mass spectral data show the presence of 1-naphthalene carboxamide in the partially purified compound. It also contains the antidepressant agent nefazodone.

\section{CONCLUSION}

The current study was aimed to characterize the antibacterial compound produced by anantagonistic Streptomyces species derived from a garden rhizosphere soil. The potential strain was recognized as Streptomyces species at genus level based on its cultural and molecular characteristics. The crude extract of this potent isolate was active against both Gram-positive, Gram-negative bacteria and fungi. It was successfully cultivated by submerged fermentation and characterization of antimicrobial compound was carried out using several modern spectroscopic techniques such as UV, IR spectrophotometery, and LC-MS spectrometry. The LC-MS compound search shows that the partially purified compound contains many compounds with various biological activities including antibacterial, antifungal, anticancer, antitumor, and antidepressant. The antimicrobial activity of the isolate must be due to the presence of imidazole, $\mathrm{N}$-trifluoroacetyl, cyclohexane, 2-nitro, stigmasterol.

\section{ACKNOWLEDGMENTS}

The authors are grateful to the management of Sathyabama University. The authors also would like to thank Biotechnology Department, Sathyabama University, for providing facilities required for the successful completion of the research work.

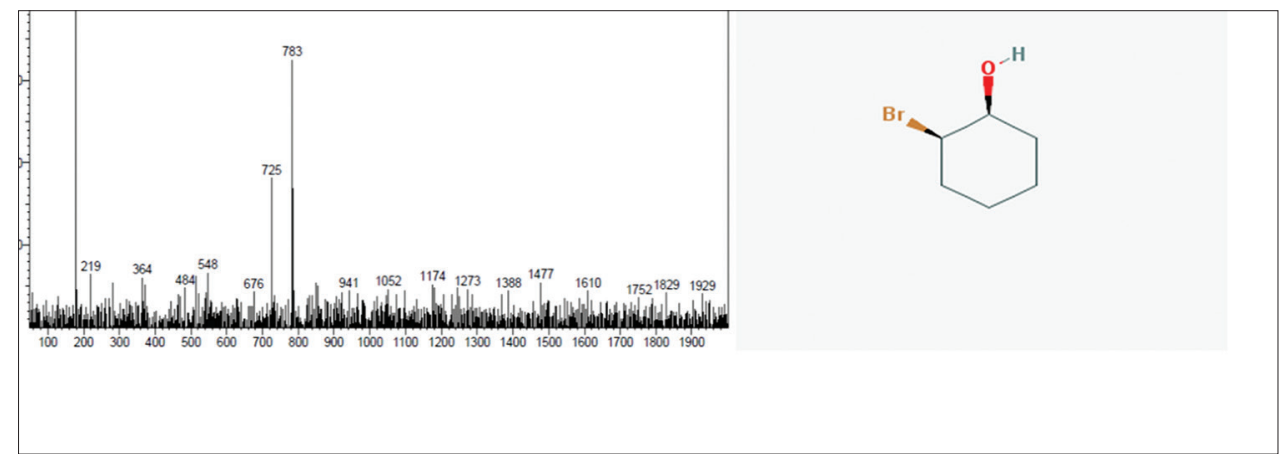

Fig. 4: Liquid chromatography-mass spectrum and chemical structure of antimicrobial compound cyclohexanol, 2-bromo-, cis- $\left(\mathrm{C}_{6} \mathrm{H}_{11} \mathrm{BrO}\right)$

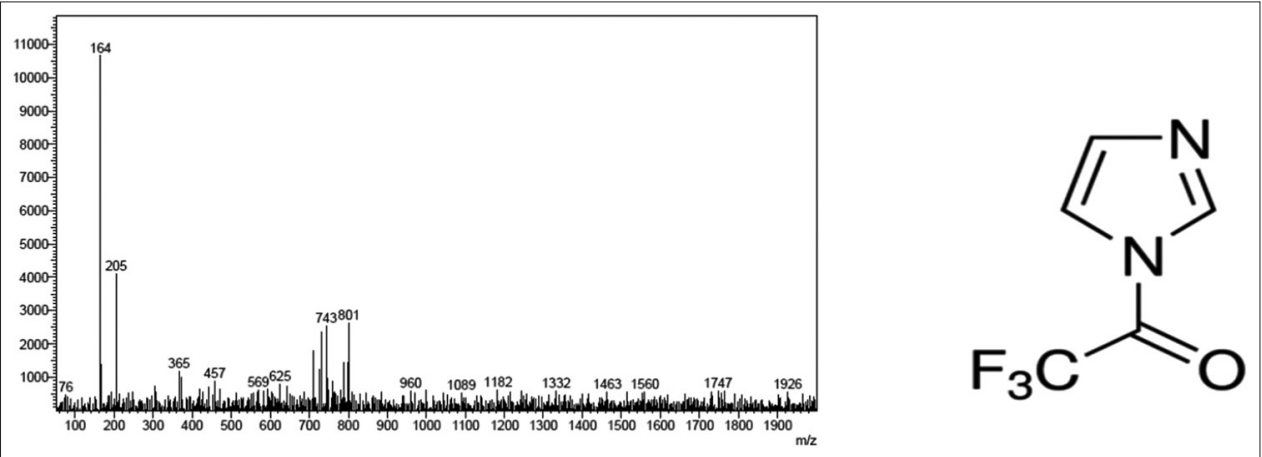

Fig. 5: Liquid chromatography-mass spectrum and chemical structure of antimicrobial compound imidazole, $N$-trifluoroacetyl $\left(C_{5} H_{3} F_{3} N_{2} 0\right)$ 


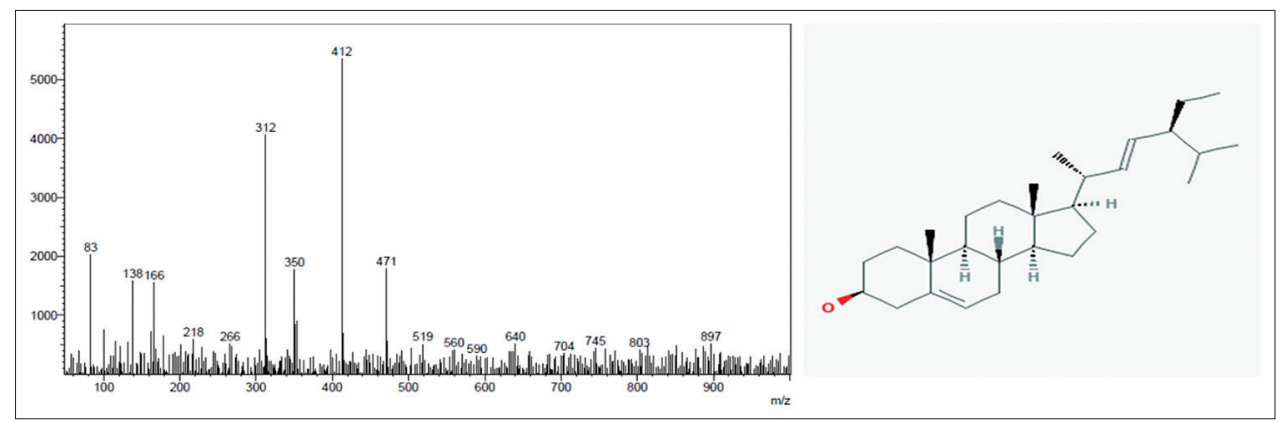

Fig. 6: Liquid chromatography-mass spectrum spectrum and chemical structure of L-antimicrobial compound stigmasterol $\left(\mathrm{C}_{29} \mathrm{H}_{48} \mathrm{O}\right)$

Table 1: Activity of identified chemical constituents in the partially purified compound by LC-MS analysis

\begin{tabular}{llll}
\hline S. No. & R.T & Name of the compounds & Activity \\
\hline 1 & 8.767 & 3-Nitrobenzenesulfonamide & Anti-cancer activity \\
2 & 12.467 & 5-Nitrofuran-2-carboxylic acid & Cytotoxicity toward human MRC-5 cells \\
3 & 19.600 & 2-Methylthiazole & anti-lymphoblastic leukemia. \\
4 & 20.933 & 4,4'-oxydiphthalic dianhydride & Anticancer \\
5 & 23.300 & Butanamide, N-(4-chlorophenyl)-3-oxo- & Anticancer \\
6 & 25.433 & Thiazolidine & Antitrypanosomal activity against Trypanosoma brucei \\
7 & 27.533 & Stigmasterol & Antibacterial and antifungal (Delia Hernández 2014) \\
8 & 35.967 & Imidazole, N-trifluoroacetyl & antimicrobials (Rokade and Sayyed, 2009) \\
9 & 39.867 & 1-naphthalene carboxamide & Cytotoxic \\
10 & 40.767 & Cyclohexanol, 2-bromo-, cis- & antidepressant \\
11 & 48.733 & Nefazodone & Antibacterial activity \\
12 & 54.767 & cyclohexane, 2-nitro & \\
\hline
\end{tabular}

LC-MS: Liquid chromatography-mass spectrum

\section{AUTHORS CONTRIBUTION}

Sudha sirkesavan: Substantial contributions to conception and design acquituons of data or analysis and interpretation and final approval of the manuscript version to be published. Usha Nandhini and Girija Vasudeven: drafting the article and revising it critically for important intellectual content.

\section{CONFLICT OF INTEREST}

we declared no conflicts of interest.

\section{REFERENCES}

1. Sathish KS, Kokati VB. In-vitro antimicrobial activity of marine Actinobacteria against multidrug resistance Staphylococcus aureus. Asian Pac J Trop Biomed 2012;2:787-92.

2. Saravana Kumar P, Duraipandiyan V, Ignacimuthu S. Isolation, screening and partial purification of antimicrobial antibiotics from soil Streptomyces sp. SCA 7. Kaohsiung J Med Sci 2014;30:435-46.

3. Kumar PS, Raj JP, Duraipandiyan V, Ignacimuthu S. Antibacterial activity of some actinomycetes from Tamil Nadu, India. Asian Pac J Trop Biomed 2012;2:936-43.

4. Solecka J, Zajko J, Postek M, Rajnisz A. Biologically active secondary metabolites from actinomycetes. Cent Eur J Biol 2012;7:373-90.

5. Raja A, Prabakarana P. Actinomycetes and drug-an overview. Am J Drug Dis Dev 2011;1:75-84.

6. Rajeswari P, Jose PA, Amiya R, Jebakumar SR. Characterization of saltern based Streptomyces $\mathrm{sp}$. and statistical media optimization for its improved antibacterial activity. Front Microbiol 2015;5:753.

7. Subramani R, Aalbersberg W. Marine actinomycetes: An ongoing source of novel bioactive metabolites. Microbiol Res 2012;10:571-80.

8. Singh LS, Sharma H, Talukdar NC. Production of potent antimicrobial agent by actinomycete, Streptomyces sannanensis strain SU118 isolated from phoomdi in Loktak Lake of Manipur, India. BMC Microbiol $2014 ; 14: 278$.

9. Berdy J. Are actinomycetes exhausted as a source of secondary metabolites. Biotechnologia 1995;7:13-34.

10. Kishore P, Mangwani N, Dash HR, Das S. Taxonomic study of antibiotic-producing marine Actinobacteria. In: Kim SK, editor. Marine Microbiology: Bioactive Compounds and Biotechnological
Applications. Weinheim: Wiley-VCH; 2013, p. 37-8.

11. Valan AM, Ignacimuthu S, Agastian P. Actinomycetes from western Ghats of Tamil Nadu with its antimicrobial properties. Asian Pac J Trop Biomed 2012;Suppl 2:S830-7.

12. Sudha S, Hemalatha R. Isolation and screening of antibiotic producing actinomycetes from garden soil of Sathyabama University, Chennai. Asian J Pharm Clin Res 2015;8:110-4.

13. Umezawa H, Kondo S, Maeda K, Okami Y, Okud T, Takeda K. Index of Antibiotics from Actinomycetes. Tokyo: University of Tokyo Press; 1967.

14. Stead P. Isolation by preparative HPLC. In: Cannell RJ, editor. Methods in Biotechnology, Natural Products Isolation. Vol. 4. Totowa, New Jersey: Humana Press; 1998. p. 165-208.

15. Bystrykh LV, Fernandez-Moreno MA, Herrema JK, Malpartida F, Hopwood DA, Dijkhuizen L. Production of action rhodinrelated "blue pigments" by Streptomyces coelicolor A3(2). J Bacteriol 1996; 178:2238-44.

16. Thirumalairaj J, Shanmugasundaram T, Sivasankari K, Natarajaseenivasan K, Balagurunathan R. Isolation, screening and characterization of potent marine Streptomyces Sp. Pm105 against antibiotic resistant pathogens. Asian J Pharm Clin Res 2015;8:439-43.

17. Slavica BI, Sandra SK, Zoran BT. UV/VIS analysis and antimicrobial activity of Streptomyces isolates. Med Biol 2005;12:44-6.

18. Parthasarathi S, Sathya S, Bupesh G, Samy RD, Mohan MR, Kumar GS, et al. Isolation and characterization of antimicrobial compound from marine Streptomyces hygroscopicus BDUS 49. World J Fish Mar Sci 2012;4:268-77.

19. Remya M, Vijayakumar R. Isolation and characterization of marine antagonistic Actinomycetes from west coast of India. Facta Univ 2008;15:13-9.

20. Nandhini SU, Bharathy PJ, Rekha S. Antifungal compounds from marine Streptomyces. Int J Pharm Pharm Sci 2014;7:207-9.

21. Saranya AV, Ravi S. Synthesis, characterization and anti-bacterial activity of pyrimidine, cyclohexanone and 1, 5-diketone derivatives of furfural chalcone. J Pharm Res 2012;5:1098-101.

22. Romero DH, Heredia VE, García-Barradas O, López ME, Pavón ES. Synthesis of imidazole derivatives and their biological activities. J Chem Biochem 2014;2:45-83.

23. Rokade YP, Sayyed RZ. Naphthalene derivatives: A new range of antimicrobials with high therapeutic value. Rasayan $\mathrm{J}$ Chem 2009;2:972-80. 